syphilis diagnosis, 42\% of studies used RPR, 24\% used VDRL, and 18\% used FTA-Abs. From 2000-2017, syphilis prevalence among MSM/TW in NA was 6.6\% (95\% CI: 5.77.4\%) and 13.3\% (95\% CI: 11.7-14.9\%) in LAC. For TW alone, prevalence was 7.1\% (95\% CI: 2.3-11.9\%) in NA and $31.7 \%$ (95\% CI: 19.1-44.4\%) in LAC. Among MSM/TW with HIV in NA, prevalence was 10.5\% (95\% CI: 7.4-13.3\%) versus $4.0 \%$ (95\% CI: $1.8-6.1 \%)$ in those without HIV. In LAC, syphilis was diagnosed in 15.1\% (95\% CI: $0-30.3 \%$ ) of MSM/TW with HIV and 12.9\% (95\% CI: 9.5-16.2\%) without HIV.

Conclusion From 2000-2017, the burden of syphilis in MSM/ TW was greater in LAC than NA. Prevalence estimates were higher in certain subgroups, including TW in LAC and MSM/ TW with HIV in NA, though these data were limited. Our results suggest a need for additional data on syphilis epidemiology with stratification by key subgroups, classification by stage of disease, and uniform diagnostic criteria to target public health strategies in the Americas.

Disclosure No significant relationships.

\section{P787 TRICHOMONAS VAGINALIS POSITIVITY RATES BY HIV STATUS AMONG WOMEN IN A CLINICAL STUDY}

${ }^{1}$ Stephanie Taylor*, ${ }^{2}$ Grace Daniel, ${ }^{3}$ Edith Torres-Chavolla, ${ }^{3}$ Charles Cooper, ${ }^{3}$ Salma Kodsi, ${ }^{2}$ Barbara Van Der Pol. 'Louisiana State University, Department of Health Sciences, New Orleans, USA; ${ }^{2}$ University of Alabama at Birmingham, Medicine/Infectious Diseases, Birmingham, USA Minor Outlying Islands; ${ }^{3}$ Becton Dickinson, Sparks, USA

\subsection{6/sextrans-2019-sti.842}

Background Numerous studies have shown that Trichomonas vaginalis (TV) infection is related to risk of HIV infection, but fewer studies have compared positivity rates based on HIV status. Further, the majority of studies that have looked at this topic have either been performed outside the US, or in HIV specialized care settings. We performed a secondary analysis of data collected for evaluation of a molecular diagnostic assay for Sexually Transmitted Infections (STI) diagnostics.

Methods Study data from patients with evaluable results obtained using a BD MAX CTGCTV study for detection of Chlamydia trachomatis (CT), Neisseria gonorrhoeae (GC) and TV were reviewed for HIV status. These women were recruited from 11 sites in the US that included STID clinics; Family Planning clinics, including Planned Parenthood clinics; $\mathrm{OB} / \mathrm{GYN}$ clinics and other clinic types. No HIV care specialty clinics participated in recruiting patients into this study. CT, GC and TV results based on HIV were compared status using $\times^{2}$ with $\alpha=0.05$.

Results HIV status data from 2339 women were available. Among HIV negative women the positivity rates for CT, GC, and TV were $6.2 \%, 1.9 \%$, and $10.3 \%$ respectively. Among HIV positive women the positivity rates for CT and TV were $2.1 \%$ and $27.1 \%$ respectively. No GC coinfection was detected among HIV positive women. For TV, the positivity rate among women with HIV [27.1\% (13/48)] was significantly higher than that of among HIV (-) women $[10.3 \%$ $(236 / 2291)](p<0.001)$.

Conclusion While women engaged in HIV specialty care may be tested for STIs routinely, as services are being pushed to more primary care settings, it is important for clinicians to be aware of the importance of STI screening among HIV (+) women, particularly for TV.

Disclosure No significant relationships.

\section{P788 COMPARISON OF VAGINAL SPECULUM AND NON- SPECULUM SPECIMENS IN THE DIAGNOSIS OF TRICHOMONAS VAGINALIS}

${ }^{1}$ Orikomaba Obunge, ${ }^{1}$ Ibinabo Oboro*, ${ }^{2}$ Nneka Onyejepu, ${ }^{1}$ Mary Alex-Wele. ${ }^{1}$ University of Port Harcourt Teaching Hospital, Medical Microbiology and Parasitology, Port Harcourt, Nigeria; ${ }^{2}$ National Institute of Medical Research, Lagos, Nigeria

\subsection{6/sextrans-2019-sti.843}

Background Trichomoniasis is the most prevalent non-viral sexually transmitted infection worldwide. In Nigeria, prevalence ranges from $5 \%$ to $15 \%$. Apart from being a key biologic indicator of sexual activity, it's been associated with the transmission of Human Immunodeficiency virus. The use of vaginal speculum is traditionally required for specimen collection. The limited application of this step in various primary health care facilities in resource-poor settings due to difficulties with sterilization of speculae among others, has grossly reduced the offer of testing for Trichomoniasis. Self-collected vaginal swab could be an acceptable alternative specimen for diagnosis of trichomoniasis in low income settings. We compared the yield of Trichomonas vaginalis from speculum and non-speculum based specimens.

Methods 500 women between 18-45 years presenting with abnormal vaginal discharge in two health care facilities in Rivers State, Nigeria, were enrolled. Three specimens were collected from each woman; two non-speculum based/noninvasive and the third, speculum based. Microscopists were trained and the procedure standardized. Wet mount microscopy was performed on all specimens within thirty minutes of collection. Three Microscopists examined each specimen independently. All infected patients were managed according to existing guidelines. Ethical approval was obtained and data analyzed using SPSS version 20.

Results Median age of participants was 31 years. Of the 500 women, 53 were infected giving an overall prevalence of $10.6 \%$. Using the speculum-based specimens, $90.5 \%$ (48) of infected women were detected while $83.0 \%$ (44) and $30.1 \%$ (16) were detected using the self-collected swab and first void urine respectively. Interestingly, first void urine was able to detect five (5) more cases that were not detected by the speculum-based method.

Conclusion In low income settings, use of self-collected swabs in combination with first void urine is likely to increase detection rate of Trichomonas vaginalis when compared with the use of vaginal speculum only.

Disclosure No significant relationships.

\section{P789 BACTERIAL VAGINOSIS MARKERS DETECTED BY BD MAX'M VAGINAL PANEL IN RELATION TO ABSENCE AND PRESENCE OF TRICHOMONAS VAGINALIS}

${ }^{1}$ Marie-Helene Tremblay*, ${ }^{2}$ Salma Kodsi, ${ }^{2}$ Charles Cooper, ${ }^{3}$ Jack Sobel. ${ }^{1} B D$ Life Sciences, Research and Development, Quebec, Canada; ${ }^{2} B D$ Life Sciences, Medical Affairs, Sparks, USA; ${ }^{3}$ Wayne State University, School of Medicine, Detroit, USA

\subsection{6/sextrans-2019-sti.844}

Background The three most frequent causes of vaginitis are bacterial vaginosis (BV), vulvovaginal candidiasis (VVC) and Trichomoniasis (TV). Within women presenting with symptoms of vaginitis, the concomitant detection of two or more pathogens is common; however, little is known about the biology of pathogen interactions during co-infections. Using a NAAT 